\title{
Caminhos e vivências de investigação acerca da saúde da população LGBT em uma capital do nordeste brasileiro
}

\section{Paths and experiences of the research process regarding the health of the LGBT population in a northeastern Brazilian capital}

\section{Caminos y experiencias de investigación sobre la salud de las personas LGBT en la capital brasileña del noreste}

\author{
Breno de Oliveira Ferreira ${ }^{1}$ \\ Edson Oliveira Pereira ${ }^{2}$ \\ Fábio Solon Tajra ${ }^{3}$ \\ Zaira Arthemisa Mesquita Araújo ${ }^{4}$ \\ Flávia Rachel Nogueira de Negreiros Freitas ${ }^{5}$ \\ José Ivo dos Santos Pedrosa ${ }^{6}$
}

RESUMO: preconceito por motivos de orientação sexual e por identidade de gênero incide na determinação social da saúde ao desenvolver processos de adoecimento e sofrimento, decorrentes do estigma social reservado à população de Lésbicas, Gays, Bissexuais, Transexuais e Travestis (LGBT) na Saúde Coletiva. Diante disso, esse trabalho trata-se de um relato de experiência que buscou analisar a condução de uma pesquisa nacional, que investigou o acesso e a qualidade da atenção integral à saúde da população de LGBT no Sistema Único de Saúde (SUS). O recorte abrange vivências e reflexões dessa produção em Teresina, Piauí. Aliado a isso, foi ressaltada a necessidade de possibilitar a reflexão sobre as atividades desenvolvidas, a fim de compreender as 1 Psicólogo; mestre em Ciências e Saúde pela Universidade Federal do Piauí (UFPI) e doutorando em Saúde, Ambiente e Sociedade (Fiocruz). Universidade Federal do Piauí (UFPI). Email: breno.oli@hotmail.com.

2 Biólogo; mestre em Saúde Coletiva e doutorando em Ciências Farmacêuticas pela Universidade de Brasília (UnB).

3 Cirurgião-dentista; docente; doutor em Saúde Coletiva pela Universidade Federal do Ceará (UFC).

4 Psicóloga; mestra em Ciências e Saúde pela Universidade Federal do Piauí (UFPI).

5 Enfermeira; mestra em Ciências e Saúde pela Universidade Federal do Piauí (UFPI).

6 Médico; docente; doutor em Saúde Coletiva pela Unicamp. 
fortalezas, fraquezas, oportunidades e ameaças na execução da pesquisa, e os resultados obtidos por meio desta análise possibilitaram uma visão detalhada da percepção dos pesquisadores sobre o processo de investigação.

Palavras-chave: Sistema Único de Saúde. Sexualidade. Equidade em saúde.

\begin{abstract}
: rejudice on the grounds of sexual orientation and gender identity focuses on the social determinants of health to develop disease processes and suffering resulting from the social stigma reserved to Lesbian, Gay, Bisexual, Transgender and Transsexual (LGBT) people in Public Health. Therefore, this work is an experience report that aimed to analyze the conduct of a national survey on the access and quality of comprehensive healthcare of the LGBT population in the Unified Health System (SUS). The outline covers experiences and reflections of this production in Teresina, Piauí. In addition, it was stressed the need to allow reflection on the activities undertaken, in order to understand the strengths, weaknesses, opportunities and threats in carrying out the research. The results obtained through this analysis enabled a detailed view of the perception of the researchers about the investigation process.
\end{abstract}

Keywords: Unified Health System, Sexuality, Health Equity.

RESUMEN: 1 prejuicio por motivos de orientación sexual e identidad de género se enfoca en los determinantes sociales de la salud para desarrollar procesos de enfermedad y sufrimiento, resultantes de un estigma social reservado a la población de Lesbianas, Gay, Bisexual, Transgénero y Transexual (LGBT) en salud pública. Por lo tanto, este trabajo es un relato de experiencia que tuvo como objetivo analizar la realización de una encuesta nacional sobre el acceso y la calidad de la atención de la salud integral de la población de los homosexuales en el Sistema Único de Salud (SUS). El esquema abarca las experiencias y reflexiones de esta producción en Teresina, Piauí. Junto a esto, se hizo hincapié en la necesidad de permitir la reflexión sobre las actividades realizadas, con el fin de entender las fortalezas, debilidades, oportunidades y amenazas en la ejecución de la investigación, y los resultados obtenidos a través de este análisis permitieron tener un panorama detallado sobre la percepción de los investigadores en el proceso de investigación.

Palabras clave: Sistema Único de Salud; sexualidad; equidad en salud.

\title{
INTRODUÇÃO
}

Trata-se de uma proposta de análise do processo de investigação relacionado à construção de conhecimentos tecno-científicos no campo da Saúde Coletiva, a partir de relatos dos pesquisadores pertencentes a um dos Grupos de Estudos e Pesquisas do Núcleo de Estudos em Saúde Pública da Universidade Federal do Piauí (NESP-UFPI).

Esta análise esteve relacionada à condução da pesquisa intitulada "Análise do acesso e da qualidade da atenção integral à saúde da população LGBT no SUS”, realizada pelo Núcleo de Estudos em Saúde Pública da Universidade de Brasília (NESP/UnB) em parceria com a FIOCRUZ/ 
PE e com as Universidades Federais da Paraíba, Piauí e Uberlândia, Universidade de São Paulo, além das Universidades Estaduais de Maringá e do Rio de Janeiro. O trabalho foi financiado pelo Departamento de Apoio à Gestão Participativa (DAGEP) da Secretaria de Gestão Estratégica e Participativa (SGEP) do Ministério da Saúde (MS), e tem sido desenvolvido desde 2014.

A intenção desta pesquisa foi mapear, identificar e compreender as dimensões do acesso aos serviços de saúde nas suas diversas possibilidades de oferta e demanda, bem como a qualidade da atenção no município de Teresina, Piauí. A partir disso, será possível articular um conjunto de ações e programas que deverão ser praticadas em todas as esferas de gestão do Sistema Único de Saúde (SUS).

A análise aqui evidenciada buscou relacionar os fatores que facilitaram ou dificultaram o processo de trabalho deste Grupo de Estudos e Pesquisas durante a execução deste tipo de investigação. Como forma de conduzir este estudo, foi formulada a seguinte questão direcionadora: Que vivências e reflexões poderão ser resgatadas a partir do desenvolvimento de uma investigação acerca da temática de saúde da população LGBT no município de Teresina?

A partir disso, discutiremos a importância do desenvolvimento de pesquisas sobre a temática de saúde da população LGBT; e as interações entre as várias etapas do trabalho e seus diferentes atores.

\section{POR QUE PESQUISAR SOBRE SAÚDE DA POPULAÇÃO LGBT?}

Nas sociedades ocidentais, dentre elas no Brasil, a sexualidade humana tem sido temática central nos mais diversos debates. Em geral, cada um deles foi permeado, em maiores ou menores proporções, pelo senso comum ou por discussões de ordem técnico-científica. Contudo, trouxeram conotações de um processo de (des)construção de sentidos e significados de cultura, subjetividade e corpo em diferentes épocas.

Para Foucault ${ }^{1}$, antes de tudo, é preciso fazer uma historiografia da sexualidade, tendo em vista a necessidade de compreender, de forma aprofundada, a sexualidade e suas práticas ao longo dos tempos. Isso se justifica pelo fato de que é importante elucidar suas formas de repressão, em que o controle dos corpos legitimou padrões de normalidade por meio de um discurso moralista e institucional até o período de liberdade sexual.

A discussão da epistemologia foucaultiana permite analisar as relações interpessoais através da tríade saber-poder-prazer, aliadas à construção de 'verdades absolutas' sobre a sexualidade' . Essas premissas inquestionáveis engendraram sentidos e condições que fazem da sexualidade movimentos narcísicos, gerando, assim, a reprodução destas sem uso reflexivo da inteligência em diferentes ambientes sociais, como nos serviços de saúde.

Em se tratando dos assuntos recorrentes, relacionados às questões sociais que tratam da 
sexualidade e do comportamento sexual, há um consenso em relação às temáticas de discriminação e violência. Quando estendemos esta discussão para a área da saúde e adentramos nas discussões acerca do acesso às ações e aos serviços básicos e especializados, parecem existir outros diversos fatores dificultadores.

Cardoso e Ferro ${ }^{2}$ apontaram que é comum ocorrer discriminação por parte de trabalhadores da saúde em relação à população LGBT e que isso está associado ao despreparo destes profissionais para lidar com as diferentes demandas desses sujeitos. De fato, o processo de formação em saúde parece não atender a esta demanda social nos mais diversos cursos. Quando se permitem discutir esta temática, estão limitados a um modelo estritamente biomédico. Esta postura poderá desencadear diversas formas de violência, seja pela abordagem inadequada dos profissionais às pessoas, seja pela ausência de dispositivos que respondam às necessidades da população LGBT nos diversos níveis de atenção à saúde.

De acordo com o Ministério da Saúde, todos os tipos de violência, seja ela velada ou explícita, devem ser considerados como fatores que impulsionam a produção de adoecimento físico e/ou mental ${ }^{3}$. Neste sentido, é preciso reunir esforços e aglutinar recursos que sejam capazes de superar esta lógica excludente que os serviços de saúde têm assumido.

No que se refere às políticas públicas de atenção integral à população LGBT, mesmo com as lutas travadas pelo movimento social, houve uma lacuna nesse campo, caracterizada pela invisibilidade e não vocalização de demandas para necessidades singulares e específicas. As primeiras ações, no início dos anos 80 , estiveram voltadas para a prevenção e o cuidado com a então epidemia HIV/ AIDS, à época fortemente conhecida como 'peste gay'4.

Foi apenas em 2004 que algumas ações foram iniciadas, como as ações de educação em saúde. Nesta oportunidade, o Governo Federal lançou o Programa 'Brasil sem Homofobia - Programa de Combate à Violência e à Discriminação contra LGBT e de Promoção da Cidadania Homossexual', que reuniu a sociedade civil e o governo em prol dos direitos da população LGBT. Tratou-se de um verdadeiro marco histórico na luta pelo direito à dignidade e pelo respeito à diferença. Isso contribuiu para a consolidação de avanços políticos, sociais e legais na área, além de promulgar a cidadania desse segmento com a equiparação de direitos e de enfrentamento à violência e à discriminação, respeitando as singularidades ${ }^{3}$.

No âmbito da saúde, o fato de instituições como a OMS/OPS terem assumido e disseminado o referencial da determinação social da saúde e doença, no sentido de elaborar estratégias globais para seus enfrentamentos, contribuiu decisivamente para que a exclusão social e a discriminação de qualquer natureza se caracterizassem como determinantes de sofrimento, adoecimento e morte. Dessa forma, os efeitos da exclusão e discriminação por questões de gênero passaram a ser considerados temas de pesquisas acadêmicas e de políticas governamentais.

$\mathrm{Na}$ busca por explicações acerca da invisibilidade de lésbicas e mulheres bissexuais, na área da Tempus, actas de saúde colet, Brasília, 11(1), 41-49, mar, 2017.

ISSN 1982-8829 
assistência integral à saúde da mulher, alguns estudos apontam que, no SUS, existem fragilidades quanto ao acolhimento desta demanda. Em geral, na busca por assistência em saúde, as mulheres sequer recebem apoio por parte dos profissionais de saúde para verbalizar suas orientações sexuais. Isso compromete a atenção integral à saúde e ocasiona uma forma de violência simbólica 5 .

Cerqueira-Santos et al..$^{6}$ apontam vários entraves vivenciados pela população LGBT no que diz respeito ao acesso e à qualidade do acesso aos serviços de saúde. No entanto, mencionam que estes são mais dramáticos nos casos das travestis e transexuais. Isso acontece não só por reivindicarem atendimento especializado para demandas que não se colocam para outros segmentos populacionais como, por exemplo, as alterações corporais associadas ao uso de hormônios e silicone, mas também pela intensidade da discriminação e exclusão que costuma incidir nos locais de atendimento em saúde.

Nesse cenário de violências, destaca-se a invisibilidade das questões de gênero nas práticas de saúde e desconsideram-se os modos de vivência da sexualidade. Em geral, tem-se atribuído a modalidade heterossexual como padrão, com implicações diretas na atenção à saúde da população LGBT. Neste sentido, a heteronormatividade age aliada a outras formas de violência estrutural, gerando um ambiente hostil e segregador nos serviços de saúde.

Para tanto, Lionço ${ }^{4}$ sinalizou a necessidade de uma política de saúde específica para esse segmento, no entanto, fundamentada na promoção da equidade, a partir da reflexão e do reconhecimento da condição de vulnerabilidade em que se encontra essa população em relação aos direitos humanos.

Em meio a esse conjunto de necessidades fortemente renegadas, levantaram-se questionamentos à luz dos direitos humanos propostos pelos movimentos sociais. Com isso, foi formulada a Política Nacional de Saúde Integral da População LGBT como resposta às demandas e especificidades emudecidas da população LGBT, seus processos de exclusão e vulnerabilidade, bem como um destaque para a centralidade estrutural na equidade em saúde, a fim de promover cidadania e efetivar uma política pública de garantia de direitos. Nesse diapasão, a Política Nacional de Saúde Integral LGBT foi aprovada pelo Conselho Nacional de Saúde em 2009 e publicada por meio da Portaria $n^{\mathrm{o}} 2.836$, de 01 de dezembro de 2011, e pactuada pela Comissão Intergestores Tripartite (CIT), conforme Resolução ${ }^{\circ} 2$ do dia 6 de dezembro de $2011^{7}$.

Apesar de todo este esforço, ainda, há muito o que se discutir. No que se refere à produção acadêmica sobre esta temática, percebemos uma infinidade de trabalhos sobre sexualidade e comportamento sexual relacionado apenas à HIV/ AIDS. Será que a demanda da população LGBT é somente essa?

A temática do acesso e qualidade da atenção também constitui um ponto que deverá ser melhor explorado nas investigações. Esta tem sido uma tendência de análise em Saúde Coletiva, tendo em vista a valorização do direito à saúde associada ao respeito dos princípios de universalidade, 
equidade e integralidade.

A partir disso, outras demandas poderão ser resgatadas e aperfeiçoadas. Contudo, podemos pontuar uma série de dificuldades no processo de condução de pesquisas.

\section{INTERAÇÕES ENTRE AS VÁRIAS ETAPAS DO TRABALHO E SEUS DIFERENTES ATORES}

A operacionalização da investigação voltada para a saúde da população LGBT na sub-estação Teresina (Piauí) exigiu a adoção de uma série de medidas importantes. Era preciso pensar nas estratégias de adequação da proposta nacional no nível local. Para isso, foi criado um Grupo Condutor (GC), formado por participantes de diversas categorias profissionais (dentre elas: medicina, enfermagem, psicologia e odontologia), tendo em vista a necessidade de estimular a apropriação temática, além de compartilhar saberes e práticas acerca do processo de desenvolvimento da pesquisa e de organização do processo de trabalho.

No que diz respeito à aproximação temática, de fato, foram apontadas fragilidades na formação em saúde quanto à atenção da população LGBT entre os participantes do grupo, conforme já havíamos mencionado anteriormente. Em geral, na academia, há pouco espaço para se dialogar sobre este assunto, quando acreditamos que, neste universo, é indispensável oportunizar uma discussão constante acerca das mais diversas questões sociais, seja por meio de estratégias de ensino, pesquisa e/ou extensão. Contudo, há pouco investimento, sobretudo, em pesquisas que tratam da saúde da população LGBT .

Neste mesmo sentido, os participantes mencionaram falhas vivenciadas por cada um deles nos mais diversos períodos de formação, quanto às estratégias de integração ensino-serviçocomunidade. Não há dúvida que se faz necessário promover uma interlocução entre o campo teórico e prático desde o início do curso, mas sabemos que nem sempre isso tem sido incentivado.

Desta forma, surgiram os primeiros questionamentos: Como superar as fragilidades da formação em saúde acerca desta temática? Que estratégias podem ser elencadas na tentativa de aproximar o universo da pesquisa à realidade vivenciada pela população LGBT?

Na nossa realidade, foi organizado um espaço de estudos e vivências acerca desta temática, como forma de superação de algumas fragilidades identificadas na formação. A cada discussão eram apontados novos assuntos que deveriam ser aprofundados e relacionados os métodos para apropriação de cada um deles.

Estes encontros possibilitaram, ainda, o fortalecimento do vínculo entre os participantes do grupo na busca por uma unidade teórico-conceitual e prática, que parecia indispensável para a continuidade do trabalho. Isso tudo porque o processo de pesquisa envolvia um "pensar e fazer 
coletivo" em torno de uma tarefa.

Havia, ainda, a necessidade de exercitar a interação e a comunicação entre os participantes do grupo, para fomentar o pensamento e a criatividade no processo de trabalho. Aliado a isso, foi ressaltada a necessidade de possibilitar a reflexão sobre as atividades desenvolvidas, particularmente, quando se tratava de compreender as fortalezas, fraquezas, oportunidades e ameaças na execução da pesquisa9.

Aliado a isso, o trabalho do Grupo Condutor favoreceu o delineamento do estudo neste município, bem como a elaboração de estratégias de atuação no campo de pesquisa. Na fase inicial, este grupo elaborou uma proposta de formação de um Grupo Operacional que envolvia a seleção de monitores. Esta seleção esteve baseada na apresentação de uma carta de intenções e relato de experiência em atividades de pesquisa e extensão, relacionadas ou não à temática do estudo a ser desenvolvido.

Com o grupo ampliado, partimos para realização de momentos teórico-conceituais com todos os participantes na intenção de promover o alinhamento teórico. Na primeira oficina, foi sugerida uma dinâmica com a intenção de perceber como cada um dos participantes percebia a abordagem à população LGBT. Em meio a uma série de situações, o grupo passou a formar uma identidade de valores que precisavam ser resgatados no tocante a sua condução.

Em outros momentos, foram convidados atores importantes para a contextualização desta questão social, além do relato de vida de cada um deles. Vale mencionar que estes momentos oportunizaram experiências singulares entre os participantes do grupo e possibilitaram a reflexão do universo que estaríamos implicados no campo de investigação.

Após a formação de uma unidade ao grupo, partimos para a operacionalização da pesquisa. $\mathrm{Na}$ seleção dos campos de investigação, buscamos contemplar diferentes territórios, agora, distribuídos em três zonas no município: zona norte; zona sul; e zona leste/sudeste. Em geral, 7 (sete) territórios foram identificados, sendo dois nas zonas norte e sul, e três na zona leste/sudeste. Os monitores foram divididos em duplas e cada uma delas contava com o apoio de dois tutores (docentes, profissionais de saúde e/ou mestrandos).

Uma vez identificados os territórios de referência para a investigação, partimos para a identificação das equipes de saúde de referência para cada uma das áreas. Após isso, foi iniciado o processo de entrevistas com os gestores dos Centros de Saúde da Família e profissionais. Cerca de 42 profissionais de saúde (médicos, enfermeiros e Agentes Comunitários de Saúde) e 7 gestores foram entrevistados. Os entrevistadores relataram ter sido bem acolhidos por cada um dos sujeitos da pesquisa. Contudo, percebemos conhecimento limitado acerca da temática do estudo, confirmando as fragilidades quanto à formação profissional. Além disso, a demanda no serviço parecia não ser frequente e isso impossibilitava, ainda mais, o contato da equipe de saúde com a população LGBT. 
É importante mencionar que houve resistência de alguns profissionais em aprofundar a discussão acerca desta temática, e isso dificultava o processo de investigação. De fato, os profissionais pareciam não ficar à vontade em tratar do assunto relacionado à saúde da população LGBT. Parecia haver uma barreira funcional, social e até mesmo cultural que afastava cada um deles da temática investigada. Mas, o serviço de saúde não deveria ser o lugar de referência para este tipo de demanda? Ser 'porta de entrada' para o sistema de saúde implica em ter limitações de acesso? Estas e outras respostas necessitariam de um maior contato com o serviço para se chegar a algumas conclusões, mas aquilo já era suficiente para mobilizar a equipe de entrevistadores e oportunizar a reflexão acerca dos assuntos que precisariam ser melhor discutidos, ainda, no espaço de ensino e serviço.

Logo após a finalização desta etapa, foi realizada oficina para análise deste processo de trabalho. Nesta oportunidade, foi utilizada a matriz SWOT para identificação das fortalezas, fraquezas, oportunidades e ameaças relacionadas ao processo de investigação, com base nos depoimentos dos pesquisadores. À luz dessa análise foi possível encontrar forças internas (fortalezas e fraquezas) e forças externas (oportunidades e ameaças). Por meio da matriz SWOT, poderíamos analisar e melhor utilizar os pontos fortes; eliminar os pontos fracos; conhecer e usufruir as oportunidades externas; e evitar as ameaças.

Os resultados obtidos por meio desta análise possibilitaram uma visão detalhada da percepção dos pesquisadores sobre o processo de investigação. Por meio disso, percebemos que a receptividade do campo abre caminhos para a formação em saúde e oferece liberdade de ação no desenvolvimento das atividades. Além disso, favorece o crescimento do futuro profissional.

Contudo, há a necessidade de envolver mais a comunidade e os profissionais dos serviços de saúde em atividades como esta. Acreditamos que isso poderá constituir um dispositivo potente para o reconhecimento das fragilidades dos serviços de saúde e estimular a mudança das práticas de trabalho.

\section{CONSIDERAÇÕES FINAIS}

Posto as diversas lacunas entre o SUS e a população LGBT, e suas evidências de que a orientação sexual e identidade de gênero são determinantes sociais de saúde, a Política LGBT surgiu como proposta de reconhecimento dos antecedentes sócio-históricos e culturais de iniquidades e exclusão social dos LGBT da agenda de saúde, e se propôs a ampliar os conhecimentos, habilidades e atitudes dos seus principais atores, a fim de dirimir os preconceitos e juízos de valor negativos em relação ao segmento, e reconhecer que assim como todo e qualquer cidadão brasileiro, os LGBT têm necessidades de saúde variadas e devem receber cuidados humanizados e pautados na integralidade em todos os níveis da atenção.

Dessa forma, o trabalho desenvolvido pelo Grupo Condutor favoreceu o delineamento dessa Política no município, bem como a elaboração de estratégias de atuação no campo de pesquisa, que 
enfrentou resistências de acesso aos profissionais, bem como das suas possibilidades de participar da pesquisa, e que de maneira geral, pode ser traduzida pelos obstáculos enfrentados pelos usuários LGBT quando procuram os serviços de saúde.

\section{REFERÊNCIAS BIBLIOGRÁFICAS}

1. Foucault M. História da sexualidade I: a vontade de saber. 1988; 13.

2. Cardoso MR, Ferro LF. Saúde e População LGBT: Demandas e Especificidades em Questão. Psicologia: ciência e profissão; 2012; 32 (3): 552-563.

3. Brasil. Ministério da Saúde. Conselho Nacional de Combate à Discriminação. Brasil sem homofobia: programa de combate à violência e à discriminação contra LGBT e promoção da cidadania homossexual. Brasília: Ministério da Saúde, 2004.

4. Lionço T. Que Direito à Saúde para a População GLBT? Considerando Direitos Humanos, Sexuais e Reprodutivos em Busca da Integralidade e da Equidade. Saúde e Sociedade; 2008; 17 (2):11-21.

5. Valadão RC, Gomes R. A homossexualidade feminina no campo da saúde: da invisibilidade à violência. PHYSIS - Revista de Saúde Coletiva, 2011; 21 (4): 1451-1467.

6. Cerqueira-Santos et al. Percepção de Usuários Gays, Lésbicas, Bissexuais e Transgêneros, Transexuais e Travestis do Sistema Único de Saúde. Revista Interamericana de Psicologia, 2010; 44 (2):235-245.

7. Brasil. Política nacional de saúde integral de Lésbicas, Gays, Bissexuais, Travestis e Transexuais. Brasília: Ministério da Saúde, 2013.

8. Soares S, Ferraz AF. Grupos operativos de aprendizagem nos serviços de saúde: sistematização de fundamentos e metodologias, Escola Anna Nery; 2007; v.11(1): 52-57.

9. Barboza R, Rocha ATS. O direito e o acesso à saúde de gays e homens que fazem sexo com homens: um olhar sobre o VIII Congresso Brasileiro de Prevenção das DST e Aids e I Congresso Brasileiro das Hepatites Virais. BIS, Bol. Inst. Saúde (Impr.), 2011; 13(2):100-110.

Artigo apresentado em 03/05/2016

Artigo aprovado em 30/06/2017

Artigo publicado no sistema em 21/09/2017 